\title{
Quasitriangularity and Enveloping Algebras for Inhomogeneous Quantum Groups
}

\author{
P. Podleśn \\ Department of Mathematics \\ University of California \\ Berkeley, CA 94720, USA
}

\begin{abstract}
Coquasitriangular universal $\mathcal{R}$ matrices on quantum Lorentz and quantum Poincaré groups are classified. The results extend (under certain assumptions) to inhomogeneous quantum groups of [10]. Enveloping algebras on those objects are described.
\end{abstract}

\section{Introduction}

Possible $R$-matrices for the fundamental representations of inhomogeneous quantum groups were found in Proposition 3.14 of [10]. In the present paper we describe universal $\mathcal{R}$ matrices for those objects (under certain assumptions which are fulfilled in the case of quantum Poincaré groups [11]). Our study will be useful in developing a simple physical model [8] of free particles on a quantum Minkowski space [11].

In Section 11 we show how to construct co(quasi)triangular $\left({ }^{*}\right.$-) bialgebras and Hopf $\left({ }^{*}-\right)$ algebras whose relations are given by means of intertwiners: we simplify and extend results of [7]. Next, in Section 2 we classify co(quasi)triangular $\left({ }^{*}\right.$-)structures $\mathcal{R}$ on quantum Lorentz groups [18]. Using the results of Sections 1 and 2, in Section 3 we classify such structures on quantum Poincaré groups [11] and also, more generally, on inhomogeneous quantum groups 10] (certain natural assumptions regarding mainly restriction of those structures to the homogeneous quantum group are made). In Section 1 we use $\mathcal{R}$ to define enveloping algebras for inhomogeneous quantum groups.

${ }^{*}$ On leave from Department of Mathematical Methods in Physics, Faculty of Physics, University of Warsaw, Hoża 74, 00-682 Warszawa, Poland

${ }^{\dagger}$ This research was supported in part by NSF grant DMS-9508597 and in part by Polish KBN grant No. 2 P301 02007 
We sum over repeated indices (Einstein's convention). We work over the field $\mathbf{C}$. We write $a \sim b$ if $a=\lambda b$ for $\lambda \in \mathbf{C} \backslash\{0\}$. If $V, W$ are vector spaces then $\tau: V \otimes W \rightarrow W \otimes V$ is given by $\tau(x \otimes y)=y \otimes x, x \in V, y \in W$. If $\mathcal{A}$ is an algebra, $v \in M_{N}(\mathcal{A}), w \in M_{K}(\mathcal{A})$, then the tensor product $v \otimes w \in M_{N K}(\mathcal{A})$ is defined by $(v \otimes w)_{i j, k l}=v_{i k} w_{j l}, i, k=1, \ldots, N$, $j, l=1, \ldots, K$. We set $\operatorname{dim} v=N$. If $\mathcal{A}$ is a ${ }^{*}$-algebra then the conjugate of $v$ is defined as $\bar{v} \in M_{N}(\mathcal{A})$ where $\bar{v}_{i j}=v_{i j}{ }^{*}, i, j=1, \ldots, N$.

Throughout the paper quantum groups $H$ are abstract objects described by the corresponding ( ${ }^{*}$-) bialgebras $\operatorname{Poly}(H)=(\mathcal{A}, \Delta)$. We denote by $\Delta, \varepsilon, S$ the comultiplication, counit and (if exists) coinverse of $\operatorname{Poly}(H)$. We say that $v$ is a representation of $H$ (i.e. $v \in \operatorname{Rep} H)$ if $v \in M_{N}(\mathcal{A}), N \in \mathbf{N}$, and $\Delta v_{i j}=v_{i k} \otimes v_{k j}, \varepsilon\left(v_{i j}\right)=\delta_{i j}, i, j=1, \ldots, N$. The conjugate of a representation and tensor product of representations are also representations. Matrix elements of representations of $H \operatorname{span} \mathcal{A}$ as a linear space. The set of nonequivalent irreducible representations of $H$ is denoted by $\operatorname{Irr} H$. If $v, w \in \operatorname{Rep} H$, then we say that $A \in M_{\operatorname{dim} w \times \operatorname{dim} v}(\mathbf{C})$ intertwines $v$ with $w$ (i.e. $\left.A \in \operatorname{Mor}(v, w)\right)$ if $A v=w A$. The dual vector space $\mathcal{A}^{\prime}$ is an algebra w.r.t. the convolution defined by $\rho * \rho^{\prime}=\left(\rho \otimes \rho^{\prime}\right) \Delta$. It has a unit $I_{\mathcal{A}^{\prime}}=\varepsilon$. For $\rho \in \mathcal{A}^{\prime}, a \in \mathcal{A}$, we set $\rho * a=(i d \otimes \rho) \Delta a, a * \rho=(\rho \otimes i d) \Delta a$.

\section{Coquasitriangular bialgebras}

In this section we discuss bialgebras $\operatorname{Poly}(G)=(\mathcal{B}, \Delta)$ defined by several fundamental representations of $G$ and intertwiners among them. We provide necessary and sufficient conditions for the existence of coquasitriangular structure $\mathcal{R}$ for $G$. Hopf and * structures are also investigated. The results generalize results of Theorem 1.4 of [7] (see also Theorem 1.1 of [18]).

Proposition 1.1 Let $\mathcal{B}$ be an algebra generated by $w_{m n}^{\alpha}, m, n=1, \ldots, d_{\alpha}, \alpha \in \mathcal{J}$ and relations $\left(0 \in \mathcal{J}, d_{0}=1\right)$

$$
\begin{gathered}
w^{\alpha} \otimes w^{0}=w^{0} \otimes w^{\alpha}=w^{\alpha}, \alpha \in \mathcal{J}, \\
W_{m}\left(w^{\alpha_{m 1}} \otimes w^{\alpha_{m 2}} \otimes \ldots w^{\alpha_{m s}}\right)=\left(w^{\beta_{m 1}} \otimes \ldots \otimes w^{\beta_{m t_{m}}}\right) W_{m}, m \in K .
\end{gathered}
$$

Then $w^{0}=\left(I_{B}\right)$ and there exists a unique $\Delta$ such that $\operatorname{Poly}(G)=(\mathcal{B}, \Delta)$ is a bialgebra and $w^{\alpha}, \alpha \in \mathcal{J}$, representations of $G$.

Proof is the same as in Theorem 1.4 of [7].

Let us recall

Definition 1.1 (cf. [1], [2], [4]) We say that $(\mathcal{B}, \Delta, \mathcal{R})$ is a coquasitriangular (CQT) bialgebra if $(\mathcal{B}, \Delta)$ is a bialgebra and $\mathcal{R} \in(\mathcal{B} \otimes \mathcal{B})^{\prime}$ satisfies

$$
\mathcal{R}(I \otimes x)=\mathcal{R}(x \otimes I)=\varepsilon(x),
$$




$$
\begin{gathered}
\mathcal{R}(x y \otimes z)=\mathcal{R}\left(x \otimes z^{(1)}\right) \mathcal{R}\left(y \otimes z^{(2)}\right), \\
\mathcal{R}(x \otimes y z)=\mathcal{R}\left(x^{(1)} \otimes z\right) \mathcal{R}\left(x^{(2)} \otimes y\right), \\
y^{(1)} x^{(1)} \mathcal{R}\left(x^{(2)} \otimes y^{(2)}\right)=\mathcal{R}\left(x^{(1)} \otimes y^{(1)}\right) x^{(2)} y^{(2)}
\end{gathered}
$$

where we have used the Sweedler's notation $\Delta(x)=x_{i}^{(1)} \otimes x_{i}^{(2)} \equiv x^{(1)} \otimes x^{(2)}$.

Remark 1.1 (cf. [5], [4], Proposition 1.3 of [7]) Let $\operatorname{Poly}(G)=(\mathcal{B}, \Delta)$ be a bialgebra and $\mathcal{R} \in(\mathcal{B} \otimes \mathcal{B})^{\prime}$. For each $v, w \in \operatorname{Rep} G$ one can define $R^{v w} \in \operatorname{Lin}\left(\mathbf{C}^{\operatorname{dim} v} \otimes \mathbf{C}^{\operatorname{dim} w}, \mathbf{C}^{\operatorname{dim} w} \otimes\right.$ $\left.\mathrm{C}^{\operatorname{dim} v}\right)$ by

$$
\left(R^{v w}\right)_{i j, k l}=\mathcal{R}\left(v_{j k} \otimes w_{i l}\right), j, k=1, \ldots, \operatorname{dim} v, i, l=1, \ldots, \operatorname{dim} w
$$

Then

$$
\begin{aligned}
& (\mathbb{1} \otimes S) R^{v_{1} w}=R^{v_{2} w}(S \otimes \mathbb{1}) \text { if } S \in \operatorname{Mor}\left(v_{1}, v_{2}\right), \\
& (S \otimes \mathbb{1}) R^{v w_{1}}=R^{v w_{2}}(\mathbb{1} \otimes S) \text { if } S \in \operatorname{Mor}\left(w_{1}, w_{2}\right),
\end{aligned}
$$

$v, w, v_{1}, w_{1} \in \operatorname{Rep} G$. Suppose that $L \subset \operatorname{Rep} G$ is such that the matrix elements of representations $v \in L$ linearly $\operatorname{span} \mathcal{B}$ (e.g. $L=\operatorname{Rep} G$ ). Consider the conditions

$$
\begin{gathered}
R^{0 v}=R^{v 0}=\mathbb{1}, v \in L, \\
R^{v_{1} \otimes v_{2}, w}=\left(R^{v_{1} w} \otimes \mathbb{1}\right)\left(\mathbb{1} \otimes R^{v_{2} w}\right), v_{1}, v_{2}, w \in L, \\
R^{v, w_{1} \otimes w_{2}}=\left(\mathbb{1} \otimes R^{v w_{2}}\right)\left(R^{v w_{1}} \otimes \mathbb{1}\right), v, w_{1}, w_{2} \in L, \\
R^{v w} \in \operatorname{Mor}(v \otimes w, w \otimes v), v, w \in L .
\end{gathered}
$$

Then $(1.10) \Leftrightarrow(1.3),(1.11) \Leftrightarrow(1.4),(1.12) \Leftrightarrow(1.5),(1.13) \Leftrightarrow(1.6)$.

Theorem 1.1 Let $\operatorname{Poly}(G)=(\mathcal{B}, \Delta)$ be a bialgebra defined in Proposition 1.1 and $R^{\alpha \beta} \in$ $\operatorname{Lin}\left(\mathbf{C}^{d_{\alpha}} \otimes \mathbf{C}^{d_{\beta}}, \mathbf{C}^{d_{\beta}} \otimes \mathbf{C}^{d_{\alpha}}\right), \alpha, \beta \in \mathcal{J}$.

The following are equivalent

1) there exists $\mathcal{R} \in(\mathcal{B} \otimes \mathcal{B})^{\prime}$ such that $(\mathcal{B}, \Delta, \mathcal{R})$ is a CQT bialgebra and $R^{\alpha \beta}=R^{w^{\alpha} w^{\beta}}$. 2)

$$
R^{0 \alpha}=R^{\alpha 0}=\mathbb{1}, \alpha \in \mathcal{J}
$$

$$
\begin{gathered}
\left(\mathbb{1} \otimes W_{m}\right) R^{\alpha_{m 1} \cdot \ldots \cdot \alpha_{m s_{m}}, \gamma}=R^{\beta_{m 1} \cdot \ldots \cdot \beta_{m t_{m}}, \gamma}\left(W_{m} \otimes \mathbb{1}\right), m \in K, \gamma \in \mathcal{J} \backslash\{0\}, \\
R^{\gamma, \beta_{m 1} \cdots \cdot \beta_{m t_{m}}}\left(\mathbb{1} \otimes W_{m}\right)=\left(W_{m} \otimes \mathbb{1}\right) R^{\gamma, \alpha_{m 1} \cdot \ldots \cdot \alpha_{m s_{m}}}, m \in K, \gamma \in \mathcal{J} \backslash\{0\}, \\
R^{\alpha \beta} \in \operatorname{Mor}\left(w^{\alpha} \otimes w^{\beta}, w^{\beta} \otimes w^{\alpha}\right), \alpha, \beta \in \mathcal{J} \backslash\{0\},
\end{gathered}
$$

where $R^{\delta_{1} \cdot \ldots \cdot \delta_{k+1}, \gamma}=\left(R^{\delta_{1} \cdot \ldots \cdot \delta_{k}, \gamma} \otimes \mathbb{1}\right)\left(\mathbb{1} \otimes R^{\delta_{k+1} \gamma}\right), R^{\gamma, \delta_{1} \ldots . \delta_{k+1}}=\left(\mathbb{1} \otimes R^{\gamma \delta_{k+1}}\right)\left(R^{\gamma, \delta_{1} \cdot \ldots \cdot \delta_{k}} \otimes \mathbb{1}\right)$, $\gamma, \delta_{1}, \ldots, \delta_{k+1} \in \mathcal{J}, k=1,2, \ldots$

Moreover, such $\mathcal{R}$ is unique. 
Remark. In special cases related statements can be found in [6], [4], [2] and Theorem 1.4 of [7]. Cf. [5].

Proof. Assume condition 1). According to Remark 1.1, (1.10)-(1.13) follow. Thus we get (1.14), (1.17). Using (1.8)-(1.9) for $S=W_{m}$ (see (1.2)) and (1.11)-(1.12), one obtains (1.15)-(1.16) and the condition 2) is proved.

Moreover, $R^{w^{\alpha} w^{\beta}}=R^{\alpha \beta}$ uniquely determine $R^{v w}$, where $v, w \in L_{0}$,

$$
L_{0}=\left\{\text { tensor products of some number of copies of representations } w^{\alpha}, \alpha \in \mathcal{J}\right\} \text {. }
$$

Using (1.7) and the fact that the matrix elements of representations from $L_{0}$ linearly span $\mathcal{B}$, the uniqueness of $\mathcal{R}$ follows. It remains to prove

$2) \Rightarrow 1$ ): Assume condition 2). Using (1.14), we can replace $\mathcal{J} \backslash\{0\}$ by $\mathcal{J}$ in (1.15)-(1.17). We define the homomorphisms $\mathcal{R}^{\beta}: \mathcal{B} \rightarrow M_{d_{\beta}}(\mathbf{C}), \beta \in \mathcal{J}$, by

$$
\left[\mathcal{R}^{\beta}\left(w_{i j}^{\alpha}\right)\right]_{k l}=R_{k i, j l}^{\alpha \beta}, \quad \alpha \in \mathcal{J}
$$

(later on we will have $\mathcal{R}_{k l}^{\beta}=\mathcal{R}\left(\cdot \otimes w_{k l}^{\beta}\right)$ ). They preserve the relations (1.1)-(1.2) due to (1.14)-(1.15). Setting $\alpha=0$ in (1.18), we show that $\mathcal{R}^{\beta}$ are unital. Setting $\beta=0$ in (1.18), one gets $\mathcal{R}_{11}^{0}=\varepsilon$ (it is true on the generators $w_{i j}^{\alpha}$ ). Hence $\mathcal{R}_{11}^{0} * \mathcal{R}_{k l}^{\beta}=\mathcal{R}_{k l}^{\beta} * \mathcal{R}_{11}^{0}=\mathcal{R}_{k l}^{\beta}$. Let

$$
\begin{aligned}
X & =\left\{x \in \mathcal{B}: W_{b_{1} \ldots b_{t_{m}}, a_{1} \ldots a_{s_{m}}}\left(\mathcal{R}_{a_{s_{m} c_{m} s_{m}}}^{\alpha_{m}} * \ldots * \mathcal{R}_{a_{1} c_{1}}^{\alpha_{m 1}}\right)(x)\right. \\
& \left.=\left(\mathcal{R}_{b_{t_{m}} d_{t_{m}}}^{\beta_{m t_{m}}} * \ldots * \mathcal{R}_{b_{1} d_{1}}^{\beta_{1 t_{1}}}\right)(x) W_{d_{1} \ldots d_{t_{m}}, c_{1} \ldots c_{s_{m}}}, m \in K\right\}
\end{aligned}
$$

It is straightforward to show that $X$ is an algebra. According to (1.16), $u_{k l}^{\gamma} \in X(k, l=$ $\left.1, \ldots, d_{\gamma}, \gamma \in \mathcal{J}\right)$. Thus $X=\mathcal{B}$. Hence there exists a linear antihomomorphism $\theta: \mathcal{B} \rightarrow \mathcal{B}^{\prime}$ such that $\theta\left(w_{k l}^{\beta}\right)=\mathcal{R}_{k l}^{\beta}, k, l=1, \ldots, d_{\beta}, \beta \in \mathcal{J}(\theta$ preserves $(1.1)-(1.2))$. Setting $\mathcal{R}(x \otimes y)=$ $[\theta(y)](x), x, y \in \mathcal{B}$, we obtain an $\mathcal{R} \in(\mathcal{B} \otimes \mathcal{B})^{\prime}$ which satisfies (1.5), (1.3). Moreover, (1.18) yields $R^{\alpha \beta}=R^{w^{\alpha} w^{\beta}}$. Let $Y=\left\{z \in \mathcal{B}: \forall x, y \in \mathcal{B} \mathcal{R}(x y \otimes z)=\mathcal{R}\left(x \otimes z^{(1)}\right) \mathcal{R}\left(y \otimes z^{(2)}\right)\right\}$. Then $Y$ is an algebra (we use (1.5)) and $w_{k l}^{\beta} \in Y$. Hence, $Y=\mathcal{B}$ and (1.4) follows. Thus (Remark 1.1) we get (1.10)-(1.12) for $L=L_{0}$. That and (1.13) for $v, w \in\left\{w^{\alpha}: \alpha \in \mathcal{J}\right\}$ (see (1.17)) give (1.13) for $L=L_{0}$, hence (Remark 1.1] (1.6) and the condition 1) is satisfied.

Remark 1.2 The unital antihomomorphism $\theta: \mathcal{B} \rightarrow \mathcal{B}^{\prime}$ introduced in the above proof exists for each CQT bialgebra (cf. [5]) and satisfies $(\theta \otimes \theta) \Delta=\Delta^{\prime} \theta$ where $\Delta^{\prime}: \mathcal{B}^{\prime} \rightarrow(\mathcal{B} \otimes \mathcal{B})^{\prime}$ is defined by $\Delta^{\prime}(\varphi)=\varphi \circ m, \varphi \in \mathcal{B}^{\prime}$, cf. Appendix of [7].

Let us now pass to the Hopf algebra structure.

Proposition 1.2 (cf. [16], Proof of Theorem 1.4.1 of [7]) Let $\operatorname{Poly}(G)=(\mathcal{B}, \Delta)$ be a bialgebra and $w, w^{\prime}, w^{\prime \prime}$ be representations of $G$. Suppose there exist $E \in \operatorname{Mor}\left(I, w \otimes w^{\prime}\right), E^{\prime} \in$ 
$\operatorname{Mor}\left(w^{\prime \prime} \otimes w, I\right)$ such that $E$ is left nondegenerate, i.e. $E_{i-}=\left(E_{i j}\right)_{j=1}^{\operatorname{dim} w^{\prime}}, i=1, \ldots, \operatorname{dim} w$, are linearly independent and $E^{\prime}$ is right nondegenerate, i.e. $E_{-k}^{\prime}=\left(E_{m k}^{\prime}\right)_{m=1}^{\operatorname{dim} w^{\prime \prime}}, k=1, \ldots, \operatorname{dim} w$, are linearly independent. Then $w^{-1}$ exists.

Proposition 1.3 (cf. [16], Proof of Theorem 1.4.1 of [7]) Let ( $\mathcal{B}, \Delta)$ be the bialgebra defined in Proposition 1.1. Suppose $\left(u^{\alpha}\right)^{-1}$ exist, $\alpha \in \mathcal{J}$. Then $(\mathcal{B}, \Delta)$ is a Hopf algebra.

A CQT bialgebra $(\mathcal{B}, \Delta, \mathcal{R})$ such that $(B, \Delta)$ is a Hopf algebra is called CQT Hopf algebra.

Proposition 1.4 (cf. Proposition 1.3.1.b of [7]) Let $\operatorname{Poly}(G)=(\mathcal{B}, \Delta)$ and $(\mathcal{B}, \Delta, \mathcal{R})$ be a CQT Hopf algebra. Then $\left(R^{v w}\right)^{-1}$ exist for any $v, w \in \operatorname{Rep} G$. Moreover $\mathcal{R}^{\prime}=\mathcal{R}(S \otimes i d)$ satisfies

$$
\mathcal{R}^{\prime}\left(x^{(1)} \otimes y^{(1)}\right) \mathcal{R}\left(x^{(2)} \otimes y^{(2)}\right)=\mathcal{R}\left(x^{(1)} \otimes y^{(1)}\right) \mathcal{R}^{\prime}\left(x^{(2)} \otimes y^{(2)}\right)=(\varepsilon \otimes \varepsilon)(x \otimes y)
$$

(i.e. $\mathcal{R}^{\prime}=\mathcal{R}^{-1}$ ) and

$$
\mathcal{R}^{\prime}\left(v_{i l} \otimes w_{j k}\right)=\left(R^{v w}\right)_{i j, k l}^{-1}, \quad i, l=1, \ldots, \operatorname{dim} v, j, k=1, \ldots, \operatorname{dim} w .
$$

We say that $(\mathcal{B}, \Delta)$ is a ${ }^{*}$-bialgebra if $(\mathcal{B}, \Delta)$ is a bialgebra, $\mathcal{B}$ is a ${ }^{*}$-algebra and

$$
(* \otimes *) \Delta(x)=\Delta\left(x^{*}\right)
$$

for $x \in \mathcal{B}$. A Hopf algebra which is a *-bialgebra is called Hopf *-algebra.

Proposition 1.5 Let $(\mathcal{B}, \Delta)$ be the bialgebra defined in Proposition 1.1. Suppose there exists an involution $\sim \mathcal{J} \rightarrow \mathcal{J}$ such that $\tilde{0}=0, d_{\tilde{\alpha}}=d_{\alpha}$ and $\tilde{W}_{m} \in \operatorname{Mor}\left(w^{\tilde{\alpha}_{m s}} \otimes \ldots \otimes\right.$ $\left.w^{\tilde{\alpha}_{m 1}}, w^{\tilde{\beta}_{m t_{m}}} \otimes \ldots \otimes w^{\tilde{\beta}_{m 1}}\right)$ where $\left(\tilde{W}_{m}\right)_{i_{t_{m}} \ldots i_{1}, j_{s_{m}} \ldots j_{1}}=\overline{\left(W_{m}\right)_{i_{1} \ldots i_{t_{m}}, j_{1} \ldots j_{s_{m}}}}$. Then there exists a unique $*: \mathcal{B} \rightarrow \mathcal{B}$ such that $(\mathcal{B}, \Delta)$ is $a^{*}$-bialgebra and $\overline{w^{\alpha}}=w^{\tilde{\alpha}}, \alpha \in \mathcal{J}$ (- was defined in the Introduction).

Proof. Uniqueness is trivial. Our assumptions imply that $z_{i j}^{\alpha}=w_{i j}^{\tilde{\alpha}}$ satisfy (1.1)-(1.2) in the conjugate algebra $\mathcal{B}^{j}$ (conjugate vector space and opposite multiplication). Therefore the desired $*$ exists (we check $*^{2}=i d$ and (1.19) on the generators $w_{i j}^{\alpha}$ ).

Proposition 1.6 (cf. the proofs of Proposition 1.3.d.ii and Theorem 1.4.6 of [7]) Let $(\mathcal{B}, \Delta, \mathcal{R})$ be a $C Q T$ bialgebra and $\operatorname{Poly}(G)=(\mathcal{B}, \Delta)$ be $a^{*}$-bialgebra. Suppose $M \subset$ Rep $G$ is such that the matrix elements of representations from $M$ generate $\mathcal{B}$ as unital algebra. The following are equivalent:

1) $\overline{\mathcal{R}\left(y^{*} \otimes x^{*}\right)}=\mathcal{R}(x \otimes y), x, y \in \mathcal{B}$

2)

$$
\overline{\left(R^{\bar{w} \bar{v}}\right)_{j i, l k}}=R_{i j, k l}^{v w}, j, k=1, \ldots, \operatorname{dim} v, i, l=1, \ldots, \operatorname{dim} w, v, w \in M .
$$

If one of the above conditions is satisfied, $(\mathcal{B}, \Delta, \mathcal{R})$ is called $C Q T^{*}$-bialgebra. If moreover $(\mathcal{B}, \Delta)$ is a Hopf algebra, $(\mathcal{B}, \Delta, \mathcal{R})$ is called CQT Hopf ${ }^{*}$-algebra (see Definition 1.1 of [Ұ]). 
Proposition 1.7 (cf. [5]) Let $\operatorname{Poly}(G)=(\mathcal{B}, \Delta)$ be a bialgebra and $\mathcal{R} \in(\mathcal{B} \otimes \mathcal{B})^{\prime}$. Suppose $M \subset \operatorname{Rep} G$ is such that the matrix elements of representations from $M$ generate $\mathcal{B}$ as unital algebra. The following are equivalent:

1) $\mathcal{R}\left(x^{(1)} \otimes y^{(1)}\right) \mathcal{R}\left(y^{(2)} \otimes x^{(2)}\right)=(\varepsilon \otimes \varepsilon)(x \otimes y), x, y \in \mathcal{B}$ (i.e. $\left.\mathcal{R}_{21}=\mathcal{R}^{-1}\right)$

2)

$$
\left(R^{v w}\right)^{-1}=R^{w v}, v, w \in M .
$$

If one of the above conditions is satisfied, we replace CQT by CT (cotriangular) in all definitions (cf. [ [ ] ).

Proof. Using (1.10)-(1.12), one can assume that (in the condition 2)) matrix elements of representations from $M$ linearly span $\mathcal{B}$. Then in 1 ) it is enough to consider $x=v_{i j}, y=w_{k l}$, $i, j=1, \ldots, \operatorname{dim} v, k, l=1, \ldots, \operatorname{dim} w, v, w \in M$. Then 1 ) is equivalent to (1.21).

\section{Quasitriangular structures on quantum Lorentz groups}

In this section we classify coquasitriangular $\left({ }^{*}\right.$-) structures on quantum Lorentz groups of [18]. For the quantum Lorentz group of [9] examples of such structures were given in [14] and Remark 7 of Section 3 of [7]. We also classify (cf. [17]) coquasitriangular $\left(^{*}{ }^{-}\right)$structures on (complex) $S L_{q}(2)$ groups and their real forms.

We first recall the definition of Hopf *-algebra corresponding to a quantum Lorentz group [18] essentially repeating the arguments of Theorem 1.1 of [18]. The bialgebra structure of $(\mathcal{A}, \Delta)$ is obtained by the construction of Proposition 1.1 with $\left\{w^{\alpha}: \alpha \in \mathcal{J}\right\}=\left\{w^{0}, w, \tilde{w}\right\}$. Here the relations (1.1) mean that $w^{0}=\left(I_{\mathcal{B}}\right)$ and the relations (1.2) are given by

$$
\begin{gathered}
(w \otimes w) E=E w^{0}, \\
(\tilde{w} \otimes \tilde{w}) \tilde{E}=\tilde{E} w^{0}, \\
E^{\prime}(w \otimes w)=w^{0} E^{\prime}, \\
\tilde{E}^{\prime}(\tilde{w} \otimes \tilde{w})=w^{0} \tilde{E}^{\prime}, \\
X(w \otimes \tilde{w})=(\tilde{w} \otimes w) X,
\end{gathered}
$$

where

$$
\tilde{E}=\tau \bar{E}, \tilde{E}^{\prime}=\bar{E}^{\prime} \tau,
$$

$E \in \operatorname{Lin}\left(\mathbf{C}, \mathbf{C}^{2} \otimes \mathbf{C}^{2}\right), E^{\prime} \in \operatorname{Lin}\left(\mathbf{C}^{2} \otimes \mathbf{C}^{2}, \mathbf{C}\right), X \in \operatorname{Lin}\left(\mathbf{C}^{2} \otimes \mathbf{C}^{2}, \mathbf{C}^{2} \otimes \mathbf{C}^{2}\right)$ satisfy

$$
\begin{gathered}
\left(E^{\prime} \otimes \mathbb{1}\right)(\mathbb{1} \otimes E)=\mathbb{1}, \\
(X \otimes \mathbb{1})(\mathbb{1} \otimes X)(E \otimes \mathbb{1})=\mathbb{1} \otimes E,
\end{gathered}
$$




$$
\tau \bar{X} \tau=\beta^{-1} X,
$$

$E^{\prime} E \neq 0, X$ is invertible, $\beta \in \mathbf{C} \backslash\{0\}$.

Warning: Our choice of $X$ may differ from the choice of [18] by a multiplicative nonzero constant.

Moreover, (2.7) implies that $E$ and $E^{\prime}$ are inverse one to another as matrices,

$$
\left(\mathbb{1} \otimes E^{\prime}\right)(E \otimes \mathbb{1})=\mathbb{1} .
$$

Hence, $E$ is left nondegenerate, $E^{\prime}$ is right nondegenerate, $w^{-1}$ exists (see Proposition 1.2). Similarly, $\left(\mathbb{1} \otimes \tilde{E}^{\prime}\right)(\tilde{E} \otimes \mathbb{1})=\mathbb{1}, \tilde{E}$ is left nondegenerate, $\tilde{E}^{\prime}$ is right nondegenerate, $\tilde{w}^{-1}$ exists. But $\left(w^{0}\right)^{-1}=w^{0}$ and Proposition 1.3 implies that $(\mathcal{A}, \Delta)$ is a Hopf algebra.

Setting $w^{\sim}=\tilde{w}, \tilde{w}^{\sim}=w, w^{0 \sim}=w^{0}$, the assumptions of Proposition 1.5 are satisfied and $(\mathcal{A}, \Delta)$ becomes a ${ }^{*}$-bialgebra where $*$ is defined by $\bar{w}=\tilde{w}$. It has the same Poincaré series as the classical $S L(2, \mathbf{C})$ group (Theorem 1.2 of [18]). We may assume that

1. $E=e_{1} \otimes e_{2}-q e_{2} \otimes e_{1}, q \in \mathbf{C} \backslash\{0, i,-i\}, X=\alpha \tau Q, Q$ is given by (13)-(19) of 18 $\left(q=-1\right.$ in (17)-(19)), $\alpha=t^{-1 / 2}$ for (13), $\alpha=(-t)^{-1 / 2}$ for (14), $\alpha=q^{1 / 2}$ for (15), $\alpha=(-q)^{1 / 2}$ for (16), $\alpha=\left(s^{2}-1\right)^{-1 / 2}$ for (17), $q=\left(p^{2}-1\right)^{-1 / 2}$ for (18), $\alpha=1 / 2$ for (19), or

2. $E=e_{1} \otimes e_{2}-e_{2} \otimes e_{1}+e_{1} \otimes e_{1}$ (in that case we set $q=1$ ), $X=\tau Q, Q$ is given by (20)-(21) of [18],

$e_{1}, e_{2}$ is the canonical basis of $\mathbf{C}^{2}$. Moreover, $\beta=t /|t|$ for (13)-(14), $\beta=q /|q|$ for (15), $\beta=-q /|q|$ for (16), $\beta=-i \operatorname{sgn} \operatorname{Im}(s)$ for $(17), \beta=\operatorname{sgn}(|p|-1)$ for (18), $\beta=1$ for (19) $-(21)$. In all cases $\beta^{4}=1$.

We shall find all $\mathcal{R} \in(\mathcal{A} \otimes \mathcal{A})^{\prime}$ such that $(\mathcal{A}, \Delta, \mathcal{R})$ is a CQT Hopf algebra. So (cf. Theorem 1.1) we need to determine $R^{w w}, R^{\bar{w} \bar{w}}, R^{\bar{w} w}, R^{w \bar{w}}$ such that 20 relations (1.15)(1.16) and 4 relations (1.17) are satisfied (we assume (1.14)). We shall use them in the following. Irreducibility of $w \otimes \bar{w}$ (see [18]) and (2.5) give $R^{w \bar{w}}=\varepsilon_{X} X, R^{\bar{w} w}=\varepsilon_{X}^{\prime} X^{-1}$, where $\varepsilon_{X}, \varepsilon_{X}^{\prime} \in \mathbf{C} \backslash\{0\}$ (cf. Proposition 1.4). Moreover, $D=R^{w w}$ and $\tilde{D}=\beta R^{\bar{w} \bar{w}}$ must satisfy $(2.11)$ and $(2.17)-(2.20)$ of [11] (with $L, \tilde{L}$ replaced by $D, \tilde{D})$, hence they are given by (2.21)-(2.22) of [11, i.e. $R^{w w}=L_{i}, R^{\bar{w} \bar{w}}=\tau \bar{L}_{j}^{-1} \tau, i, j=1,2,3,4, L_{i}=q_{i}\left(\mathbb{1}+q_{i}^{-2} E E^{\prime}\right)$, $q_{1,2}= \pm q^{1 / 2}, q_{3,4}= \pm q^{-1 / 2}$. Using (2.1), (2.3)-(2.4) of [11], we obtain $\varepsilon_{X}= \pm 1, \varepsilon_{X}^{\prime}= \pm 1$, $\beta= \pm 1$. After some calculations one gets that the 24 relations are satisfied. So we get $4 \cdot 4 \cdot 4$ $\mathcal{R}$ for $q \neq \pm 1, \beta= \pm 1,2 \cdot 2 \cdot 4 \mathcal{R}$ for $q= \pm 1, \beta= \pm 1$ and no $\mathcal{R}$ for $\beta= \pm i$.

Set $M=\{w, \bar{w}\}$. According to Proposition 11.6, we get a CQT Hopf *-algebra iff 4 relations $(1.20)$ are satisfied iff $\beta=1$ and $q_{j}=q_{i}^{-1}(4 \cdot 4 \mathcal{R}$ for $q \neq \pm 1, \beta=1,2 \cdot 4 \mathcal{R}$ for $q= \pm 1, \beta=1$, no $\mathcal{R}$ for $\beta \neq 1$ ). According to Proposition [1.7, we get CT Hopf algebra iff 4 relations (1.21) are satisfied iff $q=1, \varepsilon_{X}^{\prime}=\varepsilon_{X}(2 \cdot 2 \cdot 2 \mathcal{R}$ for $q=1, \beta= \pm 1$, no $\mathcal{R}$ 
otherwise). Clearly, we get CT Hopf ${ }^{*}$-algebra iff $q=\beta=1$ and $q_{j}=q_{i}^{-1}, \varepsilon_{X}^{\prime}=\varepsilon_{X}(2 \cdot 2 \mathcal{R}$ for $q=\beta=1$, no $\mathcal{R}$ otherwise).

Let us also recall $S L_{q}(2)$ groups [15], [1]. The bialgebra structure is obtained by the construction of Proposition 1.1 with $\left\{w^{\alpha}: \alpha \in \mathcal{J}\right\}=\left\{w^{0}, w\right\}$, the relations (1.1) mean that $w^{0}=\left(I_{\mathcal{B}}\right)$ and those of (1.2) are given by (2.1), (2.3), where $E, E^{\prime}$ are as above with $q \in \mathbf{C} \backslash\{0\}$ (only the case 1.). One gets that $(\mathcal{A}, \Delta)$ is a Hopf algebra.

We shall describe (cf. [17]) all $\mathcal{R} \in(\mathcal{A} \otimes \mathcal{A})^{\prime}$ such that $(\mathcal{A}, \Delta, \mathcal{R})$ is a CQT Hopf algebra. Due to Theorem 1.1 we should find $D=R^{w w} \in \operatorname{Mor}(w \otimes w, w \otimes w)$ satisfying 4 relations (1.15)-(1.16). This means $D=L_{i}, i=1,2,3,4$, so we get $4 \mathcal{R}$ for $q \neq \pm 1,0$, and $2 \mathcal{R}$ for $q= \pm 1$. In other words $R^{w w}= \pm L_{1}^{ \pm 1}$ where $L_{1}=q^{1 / 2}\left(\mathbb{1}+q^{-1} E E^{\prime}\right)$. We get CT Hopf algebras iff $L_{1}^{2}=\mathbb{1 1}$ (see Proposition 1.7 with $M=\{w\}$ ) iff $q=1$.

Let us pass to real forms. Then $(\mathcal{A}, \Delta)$ becomes a Hopf ${ }^{*}$-algebra where $*$ is defined by $\bar{w}=v^{c}, v=B w B^{-1}, B=\mathbb{1}$ for $S U_{q}(2), B=\operatorname{diag}(1,-1)$ for $S U_{q}(1,1), q \in \mathbf{R} \backslash\{0\}$ (cf. [15], [12], [7]), $\bar{w}=w$ for $S L_{q}(2, \mathbf{R}),|q|=1$ (cf. [12] and Proposition 1.5). For $S U_{q}(2), S U_{q}(1,1)$ we get CQT Hopf *-algebras iff $L_{1}$ is hermitian w.r.t. the canonical scalar product in $\mathbf{C}^{2}$ (see Proposition 1.6.2 with $M=\{w\}$ and the proof of Theorem 1.4.6 of [7]) iff $q>0$. For $S L_{q}(2, \mathbf{R})$ we get CQT Hopf ${ }^{*}$-algebras iff $\tau \bar{L}_{1} \tau=L_{1}$ (see Proposition 1.6.2 with $M=\{w\}$ ) iff $q=1$. So we have CT Hopf *-algebras iff $q=1$ (for each real form).

\section{Quasitriangular structures on inhomogeneous quan- tum groups}

For any Hopf algebra $\operatorname{Poly}(H)=(\mathcal{A}, \Delta)$ satisfying certain properties one can construct [10] a Hopf algebra $\operatorname{Poly}(G)=(\mathcal{B}, \Delta)$ which describes an inhomogeneous quantum group. For certain CQT Hopf algebra structures $\left(\mathcal{A}, \Delta, \mathcal{R}_{\mathcal{A}}\right)$ we find all CQT Hopf algebra structures $(\mathcal{B}, \Delta, \mathcal{R})$ such that $\mathcal{R}_{\mid \mathcal{A} \otimes \mathcal{A}}=\mathcal{R}_{\mathcal{A}}$. The ${ }^{*}$-structures and cotriangularity are studied as well. In particular we find all $\mathrm{C}(\mathrm{Q}) \mathrm{T}$ Hopf $\left(^{*}-\right)$ algebra structures on quantum Poincaré groups.

Throughout the Section $\operatorname{Poly}(H)=(\mathcal{A}, \Delta)$ is any bialgebra such that

(a) each representation of $H$ is completely reducible,

(b) $\Lambda$ is an irreducible representation of $H$,

(c) $\operatorname{Mor}(v \otimes w, \Lambda \otimes v \otimes w)=\{0\}$ for any two irreducible representations $v, w$ of $H$.

Moreover, we assume that $f_{i j}, \eta_{i} \in \mathcal{A}^{\prime}, i, j=1, \ldots, N=\operatorname{dim} \Lambda$, are given and satisfy

1. $\mathcal{A} \ni a \rightarrow \rho(a)=\left(\begin{array}{cc}f(a) & \eta(a) \\ 0 & \varepsilon(a)\end{array}\right) \in M_{N+1}(\mathbf{C})$ is a unital homomorphism,

2. $\Lambda_{s t}\left(f_{t r} * a\right)=\left(a * f_{s t}\right) \Lambda_{t r}$ for $a \in \mathcal{A}$, 
3. $R^{2}=\mathbb{1}$ where $R_{i j, s m}=f_{i m}\left(\Lambda_{j s}\right)$,

4. $(\Lambda \otimes \Lambda)_{k l, i j}\left(\tau^{i j} * a\right)=a * \tau^{k l}$ for $a \in \mathcal{A}$ where $\tau^{i j}=(R-1)_{i j, m n}\left(\eta_{n} * \eta_{m}-\eta_{m}\left(\Lambda_{n s}\right) \eta_{s}+\right.$ $\left.T_{m n} \varepsilon-f_{n b} * f_{m a} T_{a b}\right)$,

5. $A_{3} \tilde{F}=0$ where $A_{3}=\mathbb{1} \otimes \mathbb{1} \otimes \mathbb{1}-R \otimes \mathbb{1}-\mathbb{1} \otimes R+(R \otimes \mathbb{1})(\mathbb{1} \otimes R)+(\mathbb{1} \otimes R)(R \otimes$ $\mathbb{1})-(R \otimes \mathbb{1})(\mathbb{1} \otimes R)(R \otimes \mathbb{1}), \tilde{F}_{i j k, m}=\tau^{i j}\left(\Lambda_{k m}\right)$,

6. $A_{3}(Z \otimes \mathbb{1}-\mathbb{1} \otimes Z) T=0, R T=-T$ where $Z_{i j, k}=\eta_{i}\left(\Lambda_{j k}\right)$.

In particular, 4. and 5. are satisfied if $\tau^{i j}=0$.

The inhomogeneous quantum group $G$ corresponds to the bialgebra $\operatorname{Poly}(G)=(\mathcal{B}, \Delta)$ defined (cf. Corollary 3.8.a of [10]) as follows: $\mathcal{B}$ is the universal unital algebra generated by $\mathcal{A}$ and $y_{i}, i=1, \ldots, N$, with the relations $I_{\mathcal{B}}=I_{\mathcal{A}}$,

$$
\begin{gathered}
y_{s} a=\left(a * f_{s t}\right) y_{t}+a * \eta_{s}-\Lambda_{s t}\left(\eta_{t} * a\right), a \in \mathcal{A}, \\
(R-\mathbb{1})_{k l, i j}\left(y_{i} y_{j}-\eta_{i}\left(\Lambda_{j s}\right) y_{s}+T_{i j}-\Lambda_{i m} \Lambda_{j n} T_{m n}\right)=0 .
\end{gathered}
$$

Moreover, $(\mathcal{A}, \Delta)$ is a subbialgebra of $(\mathcal{B}, \Delta)$ and $\Delta y_{i}=\Lambda_{i j} \otimes y_{j}+y_{i} \otimes I$ ( $y_{i}$ were denoted by $p_{i}$ in 10 ). In particular, $\mathcal{P}=\left(\begin{array}{cc}\Lambda & y \\ 0 & I\end{array}\right)$ is a representation of $G$.

Remark 3.1 If $H$ is a matrix group, $\Lambda$ its fundamental representation and $(\mathcal{A}, \Delta)$ its corresponding Hopf algebra (generated by $\Lambda_{i j}, \Lambda_{i j}^{-1}$ ) then, assuming (a)-(c) and setting $f_{i j}=\delta_{i j} \varepsilon$, $\eta_{i}=0, T=0,(\mathcal{B}, \Delta)$ corresponds to

$$
G=H \bowtie \mathbf{R}^{N}=\left\{g=\left(\begin{array}{cc}
h & a \\
0 & 1
\end{array}\right) \in M_{N+1}(\mathbf{C}): h \in H, a \in \mathbf{R}^{N}\right\},
$$

$f(g)=f(h), y_{i}(g)=a_{i}, f \in \mathcal{A}, i=1, \ldots, N, g \in G$.

According to Corollary 3.8.b and the proof of Proposition 3.12 of [10], the bialgebra $(\mathcal{B}, \Delta)$ can be obtained by the construction of Proposition 1.1 with $\left\{w^{\alpha}: \alpha \in \mathcal{J}\right\}=\operatorname{Irr} H \cup\{\mathcal{P}\}$. Here the relations (1.1) mean that $w^{0} \equiv\left(I_{\mathcal{A}}\right)=\left(I_{\mathcal{B}}\right)$ and the relations $(1.2)$ are given by

$$
\begin{gathered}
(\mathcal{P} \otimes \mathcal{P}) R_{P}=R_{P}(\mathcal{P} \otimes \mathcal{P}) \\
(\mathcal{P} \otimes w) N_{w}=N_{w}(w \otimes \mathcal{P}), w \in \operatorname{Irr} H \\
\left(w \otimes w^{\prime}\right) S_{w w^{\prime} w^{\prime \prime}}^{\alpha}=S_{w w^{\prime} w^{\prime \prime}}^{\alpha} w^{\prime \prime}, w, w^{\prime}, w^{\prime \prime} \in \operatorname{Irr} H, \alpha=1, \ldots, c_{w w^{\prime}}^{w^{\prime \prime}} \\
\mathcal{P} i=i \Lambda \\
s \mathcal{P}=w^{0} s
\end{gathered}
$$


where

$$
R_{P}=\left(\begin{array}{cccc}
R & Z & -R \cdot Z & (R-\mathbb{1}) T \\
0 & 0 & \mathbb{1} & 0 \\
0 & \mathbb{1} & 0 & 0 \\
0 & 0 & 0 & 1
\end{array}\right), N_{w}=\left(\begin{array}{cl}
G_{w}, H_{w} \\
0, \mathbb{1}
\end{array}\right),
$$

$\left(G_{w}\right)_{i C, D j}=f_{i j}\left(w_{C D}\right),\left(H_{w}\right)_{i C, D}=\eta_{i}\left(w_{C D}\right), w \in \operatorname{Rep} H, R=G_{\Lambda}, Z=H_{\Lambda}, S_{w w^{\prime} w^{\prime \prime}}^{\alpha}$ $\left(\alpha=1, \ldots, c_{w w^{\prime}}^{w^{\prime \prime}}\right)$ form a basis of $\operatorname{Mor}\left(w^{\prime \prime}, w \otimes w^{\prime}\right), i: \mathbf{C}^{N} \rightarrow \mathbf{C}^{N} \oplus \mathbf{C}, s: \mathbf{C}^{N} \oplus \mathbf{C} \rightarrow \mathbf{C}$ are the canonical mappings. In the following we assume that $(\mathcal{A}, \Delta)$ is a Hopf algebra. Then (Proposition 3.12 of [10]) $(\mathcal{B}, \Delta)$ is also a Hopf algebra and $G_{w}^{-1}$ exist:

$$
\left(G_{w}^{-1}\right)_{A k, l B}=f_{k l}\left(w_{A B}^{-1}\right)
$$

If $(\mathcal{A}, \Delta)$ is a Hopf *-algebra then we also assume $\bar{\Lambda}=\Lambda$,

$$
f_{i j}\left(S\left(a^{*}\right)\right)=\overline{f_{i j}(a)}, \eta_{i}\left(S\left(a^{*}\right)\right)=\overline{\eta_{i}(a)}, i, j=1, \ldots, N, a \in \mathcal{A},
$$

$\tilde{T}-T \in \operatorname{Mor}\left(w^{0}, \Lambda \otimes \Lambda\right)$, where $\tilde{T}_{i j}=\overline{T_{j i}}$. Then 10$](\mathcal{B}, \Delta)$ has a unique Hopf ${ }^{*}$-algebra structure such that $(\mathcal{A}, \Delta)$ is its Hopf ${ }^{*}$-subalgebra and $y_{i}^{*}=y_{i}$.

In the following we assume $\operatorname{Mor}(I, \Lambda \otimes \Lambda) \cap \operatorname{ker}(R+\mathbb{1})=\{0\}$, i.e. $\operatorname{Mor}(I, \Lambda \otimes \Lambda) \subset$ $\operatorname{ker}(R-\mathbb{1})$. Then (using $(4.14)$ of $[10]) \tilde{T}=T$. The main result of the present paper is contained in

Theorem 3.1 Let $\operatorname{Poly}(H)=(\mathcal{A}, \Delta)$, $\operatorname{Poly}(G)=(\mathcal{B}, \Delta)$ be as above, $\left(\mathcal{A}, \Delta, \mathcal{R}_{\mathcal{A}}\right)$ be a CQT Hopf algebra such that

$$
R^{v \Lambda}=c_{v} G_{v}, R^{\Lambda v}=c_{v}^{\prime} G_{v}^{-1}, v \in \operatorname{Irr} H
$$

$c_{v}, c_{v}^{\prime} \in \mathbf{C} \backslash\{0\}$. We are interested in $C Q T$ Hopf algebra structures $(\mathcal{B}, \Delta, \mathcal{R})$ such that

$$
\mathcal{R}_{\mid \mathcal{A \otimes A}}=\mathcal{R}_{\mathcal{A}}
$$

One has:

1. Such a structure exists iff

$$
\begin{gathered}
\tau^{i j}=0, i, j=1, \ldots, N \\
R^{v \Lambda}=G_{v}, R^{\Lambda v}=G_{v}^{-1}, v \in \operatorname{Irr} H .
\end{gathered}
$$

2. Suppose (3.13)-(3.14). Then such structures are in one to one correspondence with $m \in \operatorname{Mor}\left(w^{0}, \Lambda \otimes \Lambda\right)$ satisfying

$$
\left(f_{j b} * f_{i a}\right) m_{a b}=m_{i j} \varepsilon, \quad i, j=1, \ldots, N
$$


and are determined by

$$
\begin{gathered}
R^{v w}=R^{v w} \text { for } \mathcal{R}_{\mathcal{A}}, v, w \in \operatorname{Irr} H, \\
R^{v \mathcal{P}}=N_{v}, R^{\mathcal{P} v}=N_{v}^{-1}, v \in \operatorname{Irr} H, \\
R^{\mathcal{P P}}=R_{P}+m_{P}
\end{gathered}
$$

where

$$
m_{P}=\left(\begin{array}{cccc}
0 & 0 & 0 & m \\
0 & 0 & 0 & 0 \\
0 & 0 & 0 & 0 \\
0 & 0 & 0 & 0
\end{array}\right) .
$$

3. Let $\mathcal{R}$ be as in 2. and let $(\mathcal{A}, \Delta),(\mathcal{B}, \Delta)$ be Hopf ${ }^{*}$-algebras as in the text before the Theorem. Then $(\mathcal{B}, \Delta, \mathcal{R})$ is a CQT Hopf *-algebra iff $\left(\mathcal{A}, \Delta, \mathcal{R}_{\mathcal{A}}\right)$ is a CQT Hopf *-algebra and

$$
m_{i j}=\overline{m_{j i}}, \quad i, j=1, \ldots, N .
$$

4. Let $\mathcal{R}$ be as in 2. Then $(\mathcal{B}, \Delta, \mathcal{R})$ is a CT Hopf algebra iff $\left(\mathcal{A}, \Delta, \mathcal{R}_{\mathcal{A}}\right)$ is a CT Hopf algebra and $m=0$.

Proof. Ad 1-2. Each such structure is (see Theorem 1.1) uniquely determined by $R^{v w}, R^{v \mathcal{P}}$, $R^{\mathcal{P v}}$ and $R^{\mathcal{P P}}$ satisfying (1.15)-(1.17) (we assume (1.14)), $v, w \in \operatorname{Irr} H$. Using (3.12), we get (3.16). In virtue of the properties of $\mathcal{R}_{\mathcal{A}}$ the formula (11.17) for $R^{v w}$ follows. Moreover, (1.15)(1.16) for (3.6) and $w^{\gamma}=v \in \operatorname{Irr} H$ mean $R^{v \mathcal{P}}(\mathbb{1} \otimes i)=(i \otimes \mathbb{1}) R^{v \Lambda}, R^{\mathcal{P} v}(i \otimes \mathbb{1})=(\mathbb{1} \otimes i) R^{\Lambda v}$. That and (3.11) give

$$
R^{v \mathcal{P}}=c_{v}\left(\begin{array}{c}
G_{v}, ? \\
0, ?
\end{array}\right)=c_{v} N_{v}, R^{\mathcal{P} v}=c_{v}^{\prime}\left(\begin{array}{cc}
G_{v}^{-1} & ? \\
0 & ?
\end{array}\right)=c_{v}^{\prime} N_{v}^{-1}
$$

where the second equalities follow from (1.17) for $R^{v \mathcal{P}}, R^{\mathcal{P} v}$, (3.4), the independence of $1, y_{i}$ $(i=1, \ldots, N)$ over $\mathcal{A}$ (in left and also in right module, see Corollary 3.6 and (1.4) of [10]) and the condition (c). Using (11.15)-(11.16) for (3.7) and $w^{\gamma}=v \in \operatorname{Irr} H$, one obtains $c_{v}=c_{v}^{\prime}=1$, we get (3.14), (3.17). In virtue of (1.15)-(1.16) for (3.6)-(3.7) and $w^{\gamma}=\mathcal{P}$

$$
R^{\mathcal{P P}}=\left(\begin{array}{cccc}
R & Z & -R \cdot Z & ? \\
0 & 0 & \mathbb{1} & 0 \\
0 & \mathbb{1} & 0 & 0 \\
0 & 0 & 0 & 1
\end{array}\right)=R_{P}+m_{P}
$$

for some $m \in \operatorname{Mor}\left(w^{0}, \Lambda \otimes \Lambda\right)$ where the second equality uses (1.17) for $R^{\mathcal{P P}}$, (3.3) and (3.19). Thus (3.18) follows. We set $R_{Q}=R_{P}+m_{P}=R^{\mathcal{P P}}$ and replace (3.3) by equivalent (assuming (3.5)) relation

$$
(\mathcal{P} \otimes \mathcal{P}) R_{Q}=R_{Q}(\mathcal{P} \otimes \mathcal{P}) .
$$


The relations (1.15)-(1.16) for (3.21), (3.4) and any $w^{\gamma} \in \operatorname{Irr} H \cup\{\mathcal{P}\}$ are equivalent to

$$
\begin{gathered}
\left(R_{Q} \otimes \mathbb{1}\right)\left(\mathbb{1} \otimes R_{Q}\right)\left(R_{Q} \otimes \mathbb{1}\right)=\left(\mathbb{1} \otimes R_{Q}\right)\left(R_{Q} \otimes \mathbb{1}\right)\left(\mathbb{1} \otimes R_{Q}\right), \\
\left(\mathbb{1} \otimes N_{v}\right)\left(N_{v} \otimes \mathbb{1}\right)\left(\mathbb{1} \otimes R_{Q}\right)=\left(R_{Q} \otimes \mathbb{1}\right)\left(\mathbb{1} \otimes N_{v}\right)\left(N_{v} \otimes \mathbb{1}\right), v \in \operatorname{Irr} H, \\
\left(\mathbb{1} \otimes R^{v w}\right)\left(N_{v} \otimes \mathbb{1}\right)\left(\mathbb{1} \otimes N_{w}\right)=\left(N_{w} \otimes \mathbb{1}\right)\left(\mathbb{1} \otimes N_{v}\right)\left(R^{v w} \otimes \mathbb{1}\right), v, w \in \operatorname{Irr} H .
\end{gathered}
$$

According to Proposition 3.14 of $\mathbb{1 1 0}$ and its proof, (3.22) is equivalent to $\tilde{F}=0$. Let us denote the standard basis elements in $\mathbf{C}^{\operatorname{dim} v}, v \in \operatorname{Irr} H$, by $h_{i}^{v}, i=1, \ldots, \operatorname{dim} v, e_{i}=h_{i}^{\Lambda}$ and in $\mathbf{C}$ by $f=1$. Using (3.65) of [10] and

$$
\begin{aligned}
N_{v}\left(h_{i}^{v} \otimes e_{j}\right) & =\left(G_{v}\right)_{k l, i j} e_{k} \otimes h_{l}^{v}, \\
N_{v}\left(h_{i}^{v} \otimes f\right) & =f \otimes h_{i}^{v}+\left(H_{v}\right)_{k l, i} e_{k} \otimes h_{l}^{v},
\end{aligned}
$$

we find that (3.23) on $h_{i}^{v} \otimes e_{j} \otimes e_{k}$ follows from the last formula before Proposition 3.14 in [10], on $h_{i}^{v} \otimes e_{j} \otimes f, h_{i}^{v} \otimes f \otimes e_{j}$ follows from (2.18) of [10], on $h_{i}^{v} \otimes f \otimes f$ is equivalent (using $R m=m)$ to $\tau^{i j}\left(v_{A B}\right)=0$ and (3.15) applied to $v_{A B}$ for all $i, j, A, B$. So (3.23) is equivalent to (3.13) (which implies $\tilde{F}=0$ ) and (3.15). We also get that (3.24) on $h_{i}^{v} \otimes h_{j}^{w} \otimes e_{k}$ follows from $\left(\mathbb{1} \otimes R^{v w}\right)\left(R^{v \Lambda} \otimes \mathbb{1}\right)\left(\mathbb{1} \otimes R^{w \Lambda}\right)=\left(R^{w \Lambda} \otimes \mathbb{1}\right)\left(\mathbb{1} \otimes R^{v \Lambda}\right)\left(R^{v w} \otimes \mathbb{1}\right)$ (it can be obtained using (1.13), (1.8) for $\mathcal{R}_{\mathcal{A}}-$ cf. [1], [4], (1.14) of [7]), on $h_{i}^{v} \otimes h_{j}^{w} \otimes f$ follows from the equality obtained by acting $\eta_{i}$ on (1.17) for $R^{v w}$ and using condition 1 . (see the beginning of the Section).

The relations (11.15)-(11.16) for (3.5) and $w^{\gamma} \in \operatorname{Irr} H$ follow from (1.8), (1.11) for $\mathcal{R}_{\mathcal{A}}$ while for $w^{\gamma}=\mathcal{P}$ are equivalent to

$$
\left(N_{w} \otimes \mathbb{1}\right)\left(\mathbb{1} \otimes N_{w^{\prime}}\right)\left(S_{w w^{\prime} w^{\prime \prime}}^{\alpha} \otimes \mathbb{1}\right)=\left(\mathbb{1} \otimes S_{w w^{\prime} w^{\prime \prime}}^{\alpha}\right) N_{w^{\prime \prime}}
$$

That on $h_{i}^{w^{\prime \prime}} \otimes e_{j}$ follows from (1.8), (1.11) for $\mathcal{R}_{\mathcal{A}}$, on $h_{i}^{w^{\prime \prime}} \otimes f$ follows from the equality obtained by acting $\eta_{i}$ on (3.5).

Ad 3. We need to check (1.20) for $M=\operatorname{Irr} H \cup\{\mathcal{P}\}$. For $R^{v w}, v, w \in \operatorname{Irr} H$, it is equivalent to the fact that $\left(\mathcal{A}, \Delta, \mathcal{R}_{\mathcal{A}}\right)$ is a CQT Hopf ${ }^{*}$-algebra, for $R^{\mathcal{P v}}$ and $R^{v \mathcal{P}}$ follows from (3.9), (3.10) and the properties of $\eta_{i}$, for $R^{\mathcal{P P}}$ it is equivalent (using (4.14) and the next formula of [10], $R m=m, R T=-T$ ) to (3.20).

Ad 4. We need to check (1.21) for $M=\operatorname{Irr} H \cup\{\mathcal{P}\}$. For $R^{v w}, v, w \in \operatorname{Irr} H$, it is equivalent to the fact that $\left(\mathcal{A}, \Delta, \mathcal{R}_{\mathcal{A}}\right)$ is a CT Hopf algebra, for $R^{\mathcal{P v}}$ and $R^{v \mathcal{P}}$ follows from (3.17), for $R^{\mathcal{P P}}$ it is equivalent (using $R_{P}^{2}=\mathbb{1}$ ) to $m=0$.

Remark. If the first formula of the condition 6 . is replaced by $0 \neq A_{3}(Z \otimes \mathbb{1}-\mathbb{1} \otimes Z) T \in$ Mor $(I, \Lambda \otimes \Lambda \otimes \Lambda)$ (this is allowed by [10]) then (3.22) is not satisfied and there is no CQT Hopf algebra structure on $(\mathcal{B}, \Delta)$. 
As an application we shall consider $(\mathcal{A}, \Delta)=\operatorname{Poly}(L)$ where $L$ is a quantum Lorentz group. The corresponding inhomogeneous quantum groups are called quantum Poincaré groups and are (almost) classified in [11]. The classification of $\mathrm{C}(\mathrm{Q}) \mathrm{T}$ Hopf $\left(^{*}{ }^{-}\right)$algebra structures on them is given in

Theorem 3.2 Let $\operatorname{Poly}(P)=(\mathcal{B}, \Delta)$ be the Hopf ${ }^{*}$-algebra corresponding to a quantum Poincaré group $P[11]$ described by an admissible choice of quantum Lorentz group (cases 1)-7)), $s= \pm 1, H$ and $T$.

1. Let us consider $C Q T$ Hopf algebra structures $(\mathcal{B}, \Delta, \mathcal{R})$ on $P$. One has:

a) In the cases 1) (except $s=1, t=1, t_{0} \neq 0$ - see Remark 1.8 of [1] $\mathrm{Q}$ ), 2), 3), 4) (except $s=1, b \neq 0), 5$ ) (except $s= \pm 1, t=1, t_{0} \neq 0$ ), 6), 7) each such structure is uniquely determined by

$$
R^{w w}=k L, R^{w \bar{w}}=k X, R^{\bar{w} w}=q k X^{-1}, R^{\bar{w} \bar{w}}=q k \tilde{L}
$$

and (3.17)-(3.19), where

$$
m=c m_{0}, m_{0}=\left(V^{-1} \otimes V^{-1}\right)(\mathbb{1} \otimes X \otimes \mathbb{1})(E \otimes \tau E), L=s q^{1 / 2}\left(\mathbb{1}+q^{-1} E E^{\prime}\right), \quad \tilde{L}=q \tau L \tau,
$$

where $s= \pm 1, E, E^{\prime}$ are fixed for fixed $P$ and given in [11], $k= \pm 1$ (two possible $\mathcal{R}$ for each $c \in \mathbf{C})$.

b) In the other cases there is no such structure.

2. Let $\mathcal{R}$ be as in 1 . We get CQT Hopf ${ }^{*}$-algebra iff $q=1$ (which excludes the cases 5), 6), 7)) and $c \in \mathbf{R}$.

3. Let $\mathcal{R}$ be as in 1 . We get CT Hopf algebra iff $q=1$ (which excludes the cases 5), 6), 7)) and $c=0$ (then it is also CT Hopf ${ }^{*}$-algebra).

Proof. Ad 1. We shall use Theorem 3.1, the results of [11] and Section 2. Thus $H$ is a quantum Lorentz group, $\Lambda=V^{-1}(w \otimes \bar{w}) V$ with $V_{C D, i}=\left(\sigma_{i}\right)_{C D}\left(\sigma_{0}=\mathbb{1}, \sigma_{1}, \sigma_{2}, \sigma_{3}\right.$ are the Pauli matrices), $q=\beta= \pm 1$, the assumptions about $H$ and $G$ are satisfied. Moreover, $G_{w}=\left(V^{-1} \otimes \mathbb{1}\right)(\mathbb{1} \otimes X)(L \otimes \mathbb{1})(\mathbb{1} \otimes V), G_{\bar{w}}=\left(V^{-1} \otimes \mathbb{1}\right)(\mathbb{1} \otimes \tilde{L})\left(X^{-1} \otimes \mathbb{1}\right)(\mathbb{1} \otimes V)$, where $L=s q^{1 / 2}\left(\mathbb{1}+q^{-1} E E^{\prime}\right)$ and $\tilde{L}=q \tau \overline{L^{-1}} \tau=q \tau L \tau$. The possible $\eta$ and $T$ are described in 11]. According to the results of Section 2, each CQT Hopf algebra structure on $(\mathcal{A}, \Delta)$ is uniquely characterized by $R^{w w}=\varepsilon_{L} L, R^{\bar{w} \bar{w}}=\varepsilon_{L}^{\prime} \tilde{L}, R^{w \bar{w}}=\varepsilon_{X} X, R^{\bar{w} w}=\varepsilon_{X}^{\prime} X^{-1}$, where $\varepsilon_{L}^{2}=\varepsilon_{L}^{\prime 2}=\varepsilon_{X}^{2}=\varepsilon_{X}^{\prime 2}=1$ (16 possible $\left.\mathcal{R}_{\mathcal{A}}\right)$. Using (1.8)-(1.9), (1.11)-(1.12), one gets (3.11) for $v=w, \bar{w}$ with $c_{w}=\varepsilon_{L} \varepsilon_{X}, c_{\bar{w}}=\varepsilon_{L}^{\prime} \varepsilon_{X}^{\prime}, c_{w}^{\prime}=q \varepsilon_{L} \varepsilon_{X}^{\prime}, c_{\bar{w}}^{\prime}=q \varepsilon_{X} \varepsilon_{L}^{\prime}$. In virtue of Proposition 2.1 of [11] and (1.8)-(1.9), (1.11) -(1.12) the condition (3.11) is satisfied for all $v \in \operatorname{Irr} H$.

\footnotetext{
${ }^{1}$ In the old version of Remark 1.8 of [11] one should replace $t$ by $t_{0}$ (except of expressions $t=1$ ). That $t_{0}$ is identified with $t$ of (3)-(4) of Ref. 16 of 11 .
} 
Due to Proposition 3.13.2 and Proposition 4.8 of [10], (3.13) is equivalent to $\tau^{i j}\left(w_{A B}\right)=0$, $i, j=0,1,2,3, A, B=1,2$, which means (cf. the proof of Theorem 1.6 of [11]) $\lambda=0$, which excludes the case 4$), s=1, b \neq 0$, the case 1$), s=1, t=1, t_{0} \neq 0$ and the case 5$)$, $s= \pm 1, t=1, t_{0} \neq 0$ where $t_{0} \in \mathbf{R}$ is introduced in Remark 1.8 of [11]. Moreover, (3.14) means that $\varepsilon_{X}^{\prime}=\varepsilon_{L}^{\prime}=q k, \varepsilon_{X}=\varepsilon_{L}=k$ for some $k= \pm 1$. Using Theorem 3.1.1-2, $\operatorname{Mor}\left(w^{0}, \Lambda \otimes \Lambda\right)=\mathbf{C} m_{0}$ and (3.15) for $m=m_{0}$ (it is enough to prove it on $w_{A B}, w_{A B}{ }^{*}$ when it follows from the 20 relations considered in Section 2), we get 1 .

Ad 2. We use $q=\beta=1$ (which implies $\left.q_{j}=q_{i}^{-1}\right),\left(m_{0}\right)_{i j}=\overline{\left(m_{0}\right)_{j i}}$ and Theorem 3.1.3.

Ad 3. We use $q=1$ (which implies $\varepsilon_{X}^{\prime}=\varepsilon_{X}$ ) and Theorem 3.1.4.

\section{$4 \quad$ Enveloping algebras}

In this section we study enveloping algebras of inhomogeneous quantum groups. We assume that $\left(\mathcal{A}, \Delta, \mathcal{R}_{\mathcal{A}}\right)$ and $(\mathcal{B}, \Delta, \mathcal{R})$ are CQT Hopf algebras as in Theorem 3.1.1-2 (e.g. as in Theorem 3.2.1).

We essentially follow the scheme of [12] and [13] but now we don't assume $Z=T=0$. We define $l_{j l} \in \mathcal{B}^{\prime}, j, l=1, \ldots, N,+$, by

$$
l_{j l}(x)=\mathcal{R}\left(x \otimes \mathcal{P}_{j l}\right)
$$

(in CT case $l$ corresponds to $L^{ \pm}$of [12 on the subalgebra generated by $\mathcal{P}_{a c}$ ). According to (1.5) and (1.13) for $v=w=\mathcal{P}$,

$$
\begin{aligned}
& R_{a b, c d}^{\mathcal{P} \mathcal{P}} l_{d f}\left(x^{(1)}\right) l_{c e}\left(x^{(2)}\right)=R_{a b, c d}^{\mathcal{P} \mathcal{P}} \mathcal{R}\left(x^{(1)} \otimes \mathcal{P}_{d f}\right) \mathcal{R}\left(x^{(2)} \otimes \mathcal{P}_{c e}\right) \\
& =\mathcal{R}\left(x \otimes R_{a b, c d}^{\mathcal{P P}} \mathcal{P}_{c e} \mathcal{P}_{d f}\right) \\
& =\mathcal{R}\left(x \otimes \mathcal{P}_{a c} \mathcal{P}_{b d} R_{c d, e f}^{\mathcal{P P}}\right) \\
& =\mathcal{R}\left(x^{(1)} \otimes \mathcal{P}_{b d}\right) \mathcal{R}\left(x^{(2)} \otimes \mathcal{P}_{a c}\right) R_{c d, e f}^{\mathcal{P P}} \\
& =l_{b d}\left(x^{(1)}\right) l_{a c}\left(x^{(2)}\right) R_{c d, e f}^{\mathcal{P} \mathcal{P}} \text {, }
\end{aligned}
$$

hence

$$
R_{a b, c d}^{\mathcal{P P}}\left(l_{d f} * l_{c e}\right)=\left(l_{b d} * l_{a c}\right) R_{c d, e f}^{\mathcal{P P}}, a, b, e, f=1, \ldots, N,+.
$$

Setting $l_{a b}=L_{a b}, l_{a+}=M_{a}$, and using $l_{+a}=0, l_{++}=\varepsilon, a, b=1, \ldots, N$, and (3.18), (4.2) is equivalent to

$$
\begin{gathered}
R_{a b, c d}\left(L_{d f} * L_{c e}\right)=\left(L_{b d} * L_{a c}\right) R_{c d, e f}, \\
R_{a b, c d}\left(M_{d} * L_{c e}\right)+Z_{a b, c} L_{c e}=\left(L_{b d} * L_{a c}\right) Z_{c d, e}+L_{b e} * M_{a}, \\
R_{a b, c d}\left(L_{d f} * M_{c}\right)-(R Z)_{a b, d} L_{d f}=-\left(L_{b d} * L_{a c}\right)(R Z)_{c d, f}+M_{b} * L_{a f}, \\
R_{a b, c d} M_{d} * M_{c}+Z_{a b, c} M_{c}-(R Z)_{a b, d} M_{d}+s_{a b} \varepsilon=\left(L_{b d} * L_{a c}\right) s_{c d}+M_{b} * M_{a},
\end{gathered}
$$


$a, b, e, f=1, \ldots, N$, where $s=(R-1) T+m$. Let us notice that (4.5) follows from (4.3)(4.4). Moreover, using (1.4), (1.3), $l_{a c}(x y)=l_{a b}(x) l_{b c}(y), l_{a c}(I)=\delta_{a c}, a, c=1, \ldots, N,+$, $x, y \in \mathcal{B}$. Thus $L_{a c}(I)=\delta_{a c}, M_{a}(I)=0$,

$$
\begin{gathered}
L_{a c}(x y)=L_{a b}(x) L_{b c}(y), \\
M_{a}(x y)=L_{a b}(x) M_{b}(y)+M_{a}(x) \varepsilon(y),
\end{gathered}
$$

$a, b=1, \ldots, N, x, y \in \mathcal{B}$. Also $l_{j l}\left(\mathcal{P}_{a b}\right)=\mathcal{R}\left(\mathcal{P}_{a b} \otimes \mathcal{P}_{j l}\right)=R_{j a, b l}^{\mathcal{P P}}, l_{j l}\left(w_{A B}\right)=\mathcal{R}\left(w_{A B} \otimes\right.$ $\left.\mathcal{P}_{j l}\right)=R_{j A, B l}^{w \mathcal{P}}=\left(N_{w}\right)_{j A, B l}$ (see (4.1), (1.7), (3.17), (3.8)), $a, b, j, l=1, \ldots, N,+, A, B=$ $1, \ldots, \operatorname{dim} w, w \in \operatorname{Rep} H$. Therefore

$$
\begin{gathered}
L_{j l}\left(\Lambda_{a b}\right)=R_{j a, b l}, \\
L_{j l}\left(y_{a}\right)=-(R Z)_{j a, l}, \\
L_{j l}\left(w_{A B}\right)=\left(G_{w}\right)_{j A, B l}, \\
M_{j}\left(\Lambda_{a b}\right)=Z_{j a, b}, \\
M_{j}\left(y_{a}\right)=s_{j a}, \\
M_{j}\left(w_{A B}\right)=\left(H_{w}\right)_{j A, B},
\end{gathered}
$$

$a, b, j, l=1, \ldots, N, A, B=1, \ldots, \operatorname{dim} w, w \in \operatorname{Rep} H$. It is clear that $l_{j l}$ generate a unital subalgebra of $\mathcal{B}^{\prime}$ (w.r.t. convolution $*$ ). Endowing it with $\Delta^{\prime}$ of Remark 1.2, we get a bialgebra $U$ with $l$ as its corepresentation. Adding $l_{i j}^{(m)}=l_{i j} \circ S^{m}$, one obtains a Hopf algebra $\hat{U}$ with coinverse $S^{\prime}\left(l^{(m)}\right)=l^{(m+1)}$ and corepresentations $l^{(2 k)},\left(l^{(2 k+1)}\right)^{T}, k=0,1,2, \ldots$ Acting $S^{\prime m}$ on (4.2), one obtains

$$
\begin{aligned}
R_{a b, c d}^{\mathcal{P}}\left(l_{d f}^{(2 k)} * l_{c e}^{(2 k)}\right) & =\left(l_{b d}^{(2 k)} * l_{a c}^{(2 k)}\right) R_{c d, e f}^{\mathcal{P}}, \\
R_{a b, c d}^{\mathcal{P P}}\left(l_{c e}^{(2 k+1)} * l_{d f}^{(2 k+1)}\right) & =\left(l_{a c}^{(2 k+1)} * l_{b d}^{(2 k+1)}\right) R_{c d, e f}^{\mathcal{P \mathcal { P }}} .
\end{aligned}
$$

$\hat{U}$ is called enveloping algebra of $(\mathcal{B}, \Delta)$. It can be sometimes too small. It happens e.g. in the classical case (see Remark 3.1) with $\mathcal{R}=\varepsilon \otimes \varepsilon$ when $\hat{U}=\mathbf{C} \varepsilon$. Cf. also [3].

Notice that $\left.L_{j l}\right|_{\mathcal{A}}=f_{j l},\left.M_{j}\right|_{\mathcal{A}}=\eta_{a}, l_{\mid \mathcal{A}}=\rho$. According to the proof of Theorem 1.1, there exists antihomomorphism $\theta: \mathcal{B} \rightarrow \mathcal{B}^{\prime}$ (given by $\mathcal{R}$ ) such that $\theta\left(\Lambda_{j l}\right)=L_{j l}, \theta\left(y_{j}\right)=M_{j}$, $\theta(I)=\varepsilon$. Therefore the formulae (3.60), (3.46) and (1.12) of [10] yield (4.4), (4.6) (with $s$ replaced by $(R-1) T)$ and (4.3) which give

$$
f_{b e} * \eta_{a}=R_{a b, c d} \eta_{d} * f_{c e}+Z_{a b, c} f_{c e}-\left(f_{b d} * f_{a c}\right) Z_{c d, e}
$$

(cf. (2.18) of [10]), the condition $\tau^{i j}=0$ and the last formula before Proposition 3.14 in [10]. 
Suppose $(\Lambda \otimes \Lambda) k=k w^{0}, n(\Lambda \otimes \Lambda)=w^{0} n\left(w^{0}=\left(I_{\mathcal{B}}\right)\right)$. Applying $\theta$, we get

$$
\left(L_{b d} * L_{a c}\right) k_{c d}=k_{a b} \varepsilon, n_{a b}\left(L_{b d} * L_{a c}\right)=n_{c d} \varepsilon, a, b, c, d=1, \ldots, N .
$$

Let us set $X_{l j}=L_{j l} \circ S \in \hat{U}, j, l=1, \ldots, N$.

Then

$$
\begin{gathered}
X_{i k}(x y)=X_{i j}(x) X_{j k}(y), X_{i k}(I)=\delta_{i k}, x, y \in \mathcal{B}, \\
X_{i k}(a)=f_{k i}(S(a)), a \in \mathcal{A}, i, k=1, \ldots, N .
\end{gathered}
$$

Using the last equation in the proof of Proposition 3.12 of [10], (4.7), (3.9) and (4.10), we obtain

$$
X_{i k}\left(y_{l}\right)=Z_{l k, i}
$$

Moreover, 4.15) yields

$$
k_{a b}\left(X_{a c} * X_{b d}\right)=k_{c d} \varepsilon,\left(X_{a c} * X_{b d}\right) n_{c d}=n_{a b} \varepsilon .
$$

As in the proof of Proposition 3.1.2 of [B] , there exists a unital homomorphism $X: \mathcal{B} \rightarrow$ $M_{N+1}(\mathbf{C})$ such that

$$
X=\left(\begin{array}{cc}
\left(X_{j l}\right)_{j, l=1}^{N} & \left(Y_{j}\right)_{j=1}^{N} \\
0 & \varepsilon
\end{array}\right)
$$

for some $Y_{j} \in \mathcal{B}^{\prime}$ satisfying $Y_{j}(a)=0, a \in \mathcal{A}, Y_{j}\left(y_{k}\right)=\delta_{j k}, j, k=1, \ldots, N$. Setting $X_{j+}=Y_{j}$, $X_{+j}=0, X_{++}=\varepsilon, j=1, \ldots, N$, the commutation relations among $X_{i j}, i, j=1, \ldots, N,+$, are the same as in (3.7) of [8], i.e.

$$
\left(X_{a b} * X_{c d}\right) K_{b d, s t}=K_{a c, b d}\left(X_{b s} * X_{d t}\right), a, c, s, t=1, \ldots, N,+
$$

where

$$
K=\left(\begin{array}{cccc}
R^{T} & 0 & 0 & 0 \\
0 & 0 & \mathbb{1} & 0 \\
0 & \mathbb{1} & 0 & 0 \\
0 & 0 & 0 & 1
\end{array}\right)
$$

(it is also possible to replace $K$ in $(4.20)$ by $K+n_{P}$, where $n \in \operatorname{Mor}\left(\Lambda \otimes \Lambda, w^{0}\right)$ - see (3.19), (4.19)).

Defining $X_{i j}^{(m)}=X_{i j} \circ S^{m}, i, j=1, \ldots, N,+, m=0,1,2, \ldots$, one gets a Hopf algebra $\hat{V}$ generated (as an algebra) by $l_{i j}^{(m)}$ and $X_{i j}^{(m)}$. Clearly $S^{\prime}\left(X^{(m)}\right)=X^{(m+1)} ; X^{(2 k)},\left[X^{(2 k+1)}\right]^{T}$, $k=0,1, \ldots$, are corepresentations of $\hat{V}$. Letting $S^{\prime}$ act on (4.20), one obtains

$$
\begin{aligned}
\left(X_{a b}^{(2 k)} * X_{c d}^{(2 k)}\right) K_{b d, s t} & =K_{a c, b d}\left(X_{b s}^{(2 k)} * X_{d t}^{(2 k)}\right), \\
\left(X_{c d}^{(2 k+1)} * X_{a b}^{(2 k+1)}\right) K_{b d, s t} & =K_{a c, b d}\left(X_{d t}^{(2 k+1)} * X_{b s}^{(2 k+1)}\right) .
\end{aligned}
$$

$\hat{V}$ is called enlarged enveloping algebra of $(\mathcal{B}, \Delta)$. It would be interesting to find the commutation relations between $M_{i}$ and $Y_{j}$. 


\section{Acknowledgment}

The author is grateful to Professor W. Arveson and other faculty members for their kind hospitality at UC Berkeley.

\section{References}

[1] Drinfeld, V. G., Quantum groups, in Proceedings ICM-1986, Berkeley, (1987), 798-820.

[2] Hayashi, T., Quantum groups and quantum determinants, Journal of Algebra, 152:1 (1992), 146-165.

[3] Kosiński, P. and Maślanka, P., The duality between $\kappa$-Poincaré algebra and $\kappa$-Poincaré group, hep-th 9411033 .

[4] Larson, R. G. and Towber, J., Two dual classes of bialgebras related to the concepts of "quantum group" and "quantum Lie algebra", Comm. Algebra, 19 (1991), 3295-3345.

[5] Lyubashenko, V. V., Hopf algebras and vector symmetries, Russian Math. Surveys, 41:5 (1986), 153-4. Russian original: Uspekhi Mat. Nauk, 41:5 (1986), 185-186.

[6] Majid, S., Quasitriangular Hopf algebras and Yang-Baxter equations, Int. Journal of Modern Phys. A, 5:1 (1990), 1-91.

[7] Podleś, P., Complex quantum groups and their real representations, Publ. RIMS, Kyoto Univ., 28 (1992), 709-745.

[8] Podleś, P., Solutions of Klein-Gordon and Dirac equations on quantum Minkowski spaces, q-alg 9510019, UC Berkeley preprint, PAM-654.

[9] Podleś, P. and Woronowicz, S. L., Quantum deformation of Lorentz group, Commun. Math. Phys., 130 (1990), 381-431.

[10] Podleś, P. and Woronowicz, S. L., On the structure of inhomogeneous quantum groups, hep-th 9412058, UC Berkeley preprint, PAM-631.

[11] Podleś, P. and Woronowicz, S. L., On the classification of quantum Poincaré groups, hep-th 9412059, UC Berkeley preprint, PAM-632.

[12] Reshetikhin, N. Yu., Takhtadzyan, L. A. and Faddeev, L. D., Quantization of Lie groups and Lie algebras, Leningrad Math. J., 1:1 (1990), 193-225. Russian original: Algebra $i$ analiz, 1:1 (1989), 178-206. 
[13] Schlieker, M., Weich, W. and Weixler, R., Inhomogeneous quantum groups and their quantized universal enveloping algebras, Lett. Math. Phys., 27 (1993), 217-222.

[14] Takeuchi, M., Finite dimensional representations of the quantum Lorentz group, Commun. Math. Phys., 144 (1992), 557-580.

[15] Woronowicz, S. L., Twisted $S U(2)$ group. An example of a non-commutative differential calculus, Publ. RIMS, Kyoto Univ., 23 (1987), 117-181.

[16] Woronowicz, S. L., Tannaka-Krein duality for compact matrix pseudogroups. Twisted $S U(N)$ groups, Inv. Math., 93 (1988), 35-76.

[17] Woronowicz, S.L., private information.

[18] Woronowicz, S. L. and Zakrzewski, S., Quantum deformations of the Lorentz group. The Hopf *-algebra level, Comp. Math., 90 (1994), 211-243. 